\title{
Pharmacodynamic effects of high dose lovastatin in subjects with advanced malignancies
}

\author{
Sarah A. Holstein • Howard R. Knapp • \\ Gerald H. Clamon · Daryl J. Murry • Raymond J. Hohl
}

Published online: 12 September 2006

(C) Springer-Verlag 2006

\section{Erratum to: Cancer Chemother Pharmacol 57:155-164 DOI 10.1007/s00280-005-0013-8}

Due to a processing error, numerous errors appeared in the article. The corrections are as follows:

Under the heading Introduction, the first sentence should read:

By blocking the synthesis of mevalonate, lovastatin inhibits cholesterol biosynthesis (Fig. 1).

Under the heading Methods, the first sentence should read:

Adults (age $>18$ ) with a histological diagnosis of malignancy and without an available life-prolonging therapy for that malignancy were eligible for the trial.

The legends to Figs. 1, 2, 3 and 4 should read as below:

Fig. 1 The isoprenoid biosynthetic pathway. Lovastatin inhibits HMGR, the rate-limiting step in the isoprenoid biosynthetic pathway, leading to inhibition of cholesterol synthesis. In addition, depletion of mevalonate also results in depletion of FPP and GGPP,

The online version of the original article can be found at http://dx.doi.org//10.1007/s00280-005-0013-8.

S. A. Holstein · G. H. Clamon · R. J. Hohl $(\bowtie)$

Department of Internal Medicine, University of Iowa, Iowa City, IA 52242, USA

e-mail: raymond-hohl@uiowa.edu

D. J. Murry

College of Pharmacy, University of Iowa, Iowa City, IA 52242, USA

H. R. Knapp

Research Division, Deaconess Billings Clinic, Billings, MT 59101, USA thereby preventing protein farnesylation and geranylgeranylation, catalyzed by the enzymes farnesyl protein transferase (FPTase) and geranylgeranyl protein transferases (GGPTase), respectively

Fig. 2 Comparison of peak plasma lovastatin bioactivities versus lovastatin dose. Peak plasma lovastatin bioactivities $C_{\max }(0-96)$ were measured by comparing the level of HMGR inhibition in the samples to a set of lovastatin standards as described in the Methods section

Fig. 3 Examples of changes in plasma lovastatin bioactivity levels over time with different doses of lovastatin in four different subjects. Serial samples were obtained throughout the 96-h treatment period during the first cycle. Plasma lovastatin bioactivity levels were determined as described in the Methods section

Fig. 4 Comparison of changes in cholesterol levels with lovastatin dosing (a) or peak lovastatin bioactivity levels (b). Plasma cholesterol levels at the end of the 96-h lovastatin treatment period are expressed as a percent of the control levels obtained prior to initiation of therapy

In the Results section, the first paragraph under the heading "Plasma lovastatin bioactivity", the figure citations are wrong. Fig. 1 should read Fig. 2 and Fig. 2 should be Fig. 3 .

In the Results section, the first paragraph under the heading "Plasma cholesterol levels", the figure citations are wrong. Fig. 3a should read Fig. 4a and Fig. 3b should be Fig. 4 b.

In the Discussion section the figure citations are wrong. Fig. 3 should read Fig. 4 and Fig. 1 should be Fig. 2.

Reference 29 has now been published: Leuk Res 29:527. 